\title{
BMI
}

\section{The benefits and pitfalls of pulse oximetry}

\author{
Pulse oximetry is a poor measure of hypoventilation when the concentration of inspired \\ oxygen is high
}

Over the past five years pulse oximetry has established its position as the most convenient non-invasive method of monitoring arterial saturation continuously. Widely used in operating theatres and intensive care units, it is now being increasingly used in postoperative recovery units, on general wards, in the community, and during the transport of patients.

It has several benefits as a monitoring tool: the visual detection of cyanosis is subject to considerable observer bias, ${ }^{1}$ and cyanosis is detected in only $95 \%$ of patients by experienced observers when the arterial saturation falls to $89 \% .^{2}$ In contrast, plethoric or polycythaemic patients may appear cyanosed despite adequate arterial oxygen tensions. Most deaths and major neurological sequelae in anaesthetic and perioperative practice result in some way from hypoxia, ${ }^{3}$ and clearly a non-invasive monitor of arterial saturation that is applicable to a wide range of patients has a valuable role.

But pulse oximetry is not a foolproof method: it combines the inaccuracies of this method of measurement of arterial saturation and the limitations of arterial saturation as an assessment of the cardiorespiratory status of the patient. Pulse oximeters measure the differential absorption of two wavelengths of light by total haemoglobin and oxyhaemoglobin. The pulsatile component of the signal represents the arterial absorption and forms only a small proportion of the total. The signal is thus very susceptible to "noise", especially in association with poor peripheral circulation ${ }^{4}$ and movement (for example, during transportation). A signal is possible in the absence of an arterial pulse, ${ }^{5}$ and fluctuations in ambient light can produce false pulsatile signals. ${ }^{6}$ Methaemoglobin absorbs similarly in the red and infrared bands, and its presence biases the oximeter reading to $85 \%$, irrespective of the degree of oxygenation. ${ }^{7}$ Carboxyhaemoglobin causes the pulse oximeter to overread and in carbon monoxide poisoning the displayed saturation was found to equal the sum of oxyhaemoglobin and carboxyhaemoglobin values over a range of carboxyhaemoglobin from $1 \%$ to $30 \% .{ }^{8}$ Nail polish has a variable effect depending on its colour and type. Fetal and adult haemoglobin have similar absorption spectra, so preserving the accuracy of the measurement in the neonate, ${ }^{9}$ and the commonest serum pigment, bilirubin, has little effect on the readings. ${ }^{10}$

No absolute method for calibrating pulse oximeters yet exists, and manufacturers make use of "look up" tables obtained by comparing pulse oximeter readings with multi- wavelength oximeter measurements of saturation in arterial blood samples from hypoxaemic volunteers. ${ }^{11}$ As obtaining readings during profound hypoxaemia is clearly more difficult, less confidence can be placed on readings at low saturations. ${ }^{12}$ Despite these limitations the pulse oximeter is nevertheless very useful for detecting the presence of serious hypoxaemia, and a sustained trend of falling saturation is always clinically important even if the precision of individual readings is poor.

Pulse oximeters require a pulsatile flow at the site of measurement to function and thus give a very crude indication of peripheral perfusion. The presence of pulsation, however, in no way guarantees adequacy of cardiac output, arterial blood pressure, or cardiac rhythm, and pulse oximetry cannot replace the appropriate monitoring of these variables. The relation between arterial oxygen tension $\left(\mathrm{PaO}_{2}\right)$ and arterial saturation is described by the sigmoid oxyhaemoglobin dissociation curve, and the saturation becomes a useful indicator of $\mathrm{PaO}_{2}$ only when it has fallen below $90 \%\left(\mathrm{a} \mathrm{PaO}_{2}\right.$ of about $8 \mathrm{kPa}$ ). Pulse oximetry cannot be used to monitor $\mathrm{PaO}_{2}$ when the upper level of $\mathrm{PaO}_{2}$ is critical (for example, in neonates).

A serious pitfall in the use of saturation monitoring is shown by the case report in this journal recently, which revealed a misunderstanding of the basic physiology of respiratory failure. ${ }^{13}$ Alveolar hypoventilation is the main form of respiratory failure postoperatively and results from combinations of central respiratory depression, muscular weakness, and upper airways obstruction. As arterial carbon dioxide tension rises so does alveolar carbon dioxide tension $\left(\mathrm{PCO}_{2}\right)$; alveolar $\mathrm{Po}_{2}$ falls, leading to arterial hypoxaemia. If the patient is breathing room air then the saturation will fall early and is a reasonably sensitive indicator of hypoventilation. The situation is different if the patient is receiving supplemental oxygen. The alveolar $\mathrm{Po}_{2}$ will now be much higher and alveolar $\mathrm{PCO}_{2}$ will have to rise much further before hypoxaemia sufficient to produce measurable desaturation occurs.

The failure to detect hypoventilation in such a patient is not a failure of pulse oximetry as such but an example of a false sense of security generated by a single physiological variable being within safe limits. The same caveat applies to the use of pulse oximetry to detect hypoventilation under anaesthesia, when patients routinely receive high fractional inspired oxygen concentrations.

Although pulse oximetry may be "the most significant 
technological advance ever made in the monitoring of the well-being and safety of patients during anaesthesia, recovery and critical care," 14 it must be understood that a normal reading of saturation in the presence of an increased inspired oxygen concentration gives no information about the adequacy of ventilation. Falls in saturation under these circumstances will occur late and are non-diagnostic. Under no circumstances should the pulse oximeter be relied on as the sole monitor to detect such events as oesophageal intubation, cardiac arrest, breathing system disconnections, or failure of the oxygen supply.

PETER HUTTON Professor

TOM CLUTTON-BROCK Senior lecturer

Department of Anaesthesia and Intensive Care,

University of Birmingham, Queen Elizabeth Hospital,

Birmingham B15 2TH
1 Conroe JH, Bothello $\mathrm{S}$. The unreliability of cyanosis in the recognition of arterial anoxemia. $A m \mathcal{f}$ Med Sci 1947;214:1-9.

2 Kelman GR, Nunn JF. Clinical recognition of hypoxaemia under fluorescent lamps. Lancet 1966;i:1400-3.

3 Utting JE. Pitfalls in anaesthetic practice. Br F Anaesth 1987;59:877-90.

4 Tremper KK, Hufstedler SM, Barker SJ, Adams AL, Wong DH, Zaccari J, et al. Accuracy of pulse oximeter in the critically ill adult: effect of temperature and haemodynamics. Anesthesiology 1985;65:A175.

5 Norley I. Erroneous activation of the pulse oximeter. Anaesthesia 1987;42:1116.

6 Ralston AC, Webb RK, Runciman WB. Potential estros in pulse oximetry, III. Effects of interference, dyes, dyshaemoglobins and other pigments. Anaesthesia 1991;46:291-5.

7 Reider HU, Frei FJ, Zbinden AM, Thomson DA. Pulse oximetry in methaemoglobinaemia. Anaesthesia 1989;44:326-7.

8 Vegfors M, Hennmarket C. Carboxyhaemoglobin and pulse oximetry. Br $\mathcal{A}$ Anaesth 1991;66:625-6. Dziedzic K, Vidayasagar D. Pulse oximetry in neonatal intensive care. Clin Perinatol 1989;16: 177-97.

10 Veyckemans F, Baele P, Guillamme JE, Williams E, Robert A, Clerbaux T. Hyperbilirubinaemia does not interfere with haemoglobin saturation measured by pulse oximetry. Anesthesiology 1989;70:118-22.

11 Ralston AC, Webb RK, Runciman WB. Potential errors in pulse oximetry. I. Pulse oximetry valuation. Anaesthesia 1991;46:202-6.

12 Severinghaus JW, Naifeh KH, Koh SO. Errors in 14 pulse oximeters during profound hypoxia. f Clin Monitoring 1989;5:72-81.

13 Davidson JAH, Hosie HE. Limitations of pulse oximetry: respiratory insufficiency-a failure of detection. BMF 1993;307:372-3.

14 Severinghaus JW, Astrup PB. History of blood-gas analysis. VI. Oximetry. $f$ Clin Monit 1986;2:270-88.
Patients treated in intensive care units may develop various psychiatric disorders, which become manifest either in the unit or after discharge. In many cases the psychiatric disorder, notably delirium, results from physical complications of the underlying surgical or medical illness. In others it results from the emotional impact of the illness, while in those patients admitted after accidents or self inflicted injuries the psychiatric disorder may already have been present.

In another group of patients environmental factors may be important. The first generation of intensive care units was thought to have the potential to cause psychological harm, with the unique environment of the unit precipitating psychiatric disorders in some patients. ${ }^{12}$ Several aspects were implicated, including sleep deprivation, constant noise, sensory monotony, the presence of monitoring equipment, and the lack of orientating cues. Some authors questioned the influence of environmental factors, ${ }^{3}$ whose role has never been adequately clarified. Perhaps this reflects a failure to distinguish between true delirium, which always has an organic cause, and an acute functional psychosis in which environmental factors play a more important role and which resolves when the patient is moved from the intensive care unit to the less intimidating environment of a general ward. ${ }^{4}$

Nevertheless, these early observations have affected the design of intensive care units and nursing and medical practice within them. Efforts are now made to reduce noise; encourage more human contact; provide external windows, visible clocks, and calendars; and ensure adequate sleep with a normal day-night cycle.

Until recently little was known about long term outcome, but studies of patients who have been in exceptionally threatening or catastrophic accidents have shown them to be prone to prolonged psychological reactions, particularly post-traumatic stress disorder and other phobic anxiety syndromes.

The characteristic features of post-traumatic stress disorder include intrusive memories of the original accident (flashbacks), recurrent nightmares, emotional numbing, autonomic hyperarousal, and avoidance of stimuli reminiscent of the original trauma. Patterson and colleagues observed that $30 \%$ of patients with major burns met the full DSM-III criteria for post-traumatic stress disorder at some time during their admission, but none did at the time of their discharge from hospital. They concluded that in patients with burns the disorder generally resolves without specific intervention. ${ }^{5}$ Conflicting evidence has been reported by two other studies, which suggested that the prevalence of post-traumatic stress disorder increases after discharge because survivors often develop new symptoms once they leave hospital. ${ }^{7}$ These observations are more in keeping with the view that the disorder is a delayed response to severe stress and suggest that longitudinal surveillance is needed to detect new cases and provide treatment. Post-traumatic stress disorder occurs in an estimated $10 \%$ of people with multiple injuries after road traffic accidents, but it is often not recognised and therefore not treated. ${ }^{8}$

More long term studies of patients treated in intensive care units are required to determine the prevalence of these disorders and their natural course. Aetiological factors also need to be understood. Current opinion considers the nature of the trauma to be of primary importance, but do patients' personalities have a part to play, and are there any features of modern intensive care environments that predispose to long term psychological problems?

Following up patients is difficult because they may be referred from a wide area and they are often not seen subsequently by the medical team responsible for their treatment in the intensive care unit. General practitioners are best placed to evaluate psychological symptoms once patients have left hospital, and they should be alert to the possible development of new symptoms many months after the original incident. Serious problems may be averted if doctors take a proactive approach, encouraging patients to discuss their emotional response to their accident or illness and their experiences in the intensive care unit. Direct questions need to be asked to uncover symptoms of the post-traumatic stress disorder and the less dramatic phobic anxiety disorders that may occur in accident victims.

Detection is certainly worthwhile because patients can be helped by treatment with antidepressant drugs and various psychological techniques, including psychotherapy, desensitisation, and cognitive therapy. ${ }^{910}$ Clinical experience 